\title{
Estandarización de una formulación de panelitas de leche: estudio preliminar
}

\author{
Standardization of a formulation of panelitas de leche: preliminary study. \\ José Rafael Ortiz-Álvarez; Amanda Cortés-Jiménez²; Juan Sebastián Ramírez-Navas ${ }^{3}$
}

1 Ingeniero de Alimentos, Escuela de Ingeniería de Alimentos, Universidad del Valle, Cali, Colombia. jrafael555@hotmail.com.

2 Ingeniera de Alimentos, Escuela de Ingeniería de Alimentos, Universidad del Valle, Cali, Colombia. amandita1307@hotmail.com.

3 Profesor Asistente, Ph.D., Grupo GIPAB, Escuela de Ingeniería de Alimentos, Universidad del Valle, Calle 13 No 100-00, Edificio 338, Espacio 2022, Cali, Colombia. juan.sebastian.ramirez@correounivalle.edu.co.

Citar: ORTIZ-ALVAREZ, J.; CORTÉS-JIMÉNEZ, A.; RAMÍREZ-NAVAS, J. 2017. Estandarización de una formulación de panelitas de leche: estudio preliminar. Rev. Cienc. Agr. 34(1): 64-73. doi: http://dx.doi.org/10.22267/rcia.173401.63.

Recibido: Mayo 10 de $2016 . \quad$ Aceptado: Agosto 8 de 2016.

\section{RESUMEN}

Las panelitas de leche (PDL) son un tipo de caramelo perteneciente a la familia de los dulces de leche. El objetivo de esta investigación fue obtener una formulación de PDL con lactosuero. Se realizaron ocho experimentos, todos ellos unifactoriales. Se estudió el efecto de diferentes materias primas y las condiciones del proceso sobre las características sensoriales de las PDL. Previamente, se obtuvo un perfil sensorial de PDL comerciales que sirvió de referencia al momento de evaluar los productos obtenidos en cada experimento. Se obtuvieron PDL similares a las comerciales utilizando harina de maíz, sustituyendo un $4 \%$ de azúcar por panela, con lactosuero diluido al 50\% en peso y proporciones de reemplazo de leche por lactosuero del 1:0,25; 1:0,538 o 1:1. Teniendo como condiciones de proceso una temperaturas de $90^{\circ} \mathrm{C}$ con un tiempo de cocción estimado de 150 minutos y una concentración final de $79^{\circ} \mathrm{Bx}$.

Palabras clave: Dulce de leche, lactosuero, postre lácteo, estandarización, condiciones de proceso.

\begin{abstract}
"Panelitas de leche" (PDL) are a candy-like belonging to the family of Dulce de Leche. The objective of this study was to produce a PDL formulation with whey added. Eight experiments were performed, all mono-factorials. The effects of different raw materials and the processing conditions on the sensory characteristics of the PDL were
\end{abstract}


studied. A sensory profile of commercial PDL was previously obtained to use as a reference when evaluating the food products obtained after each experiment. The best formulations of PDL - similar to commercial PDL- were obtained using corn flour, substituting $4 \%$ of sugar for panela, whey diluted by $50 \%$ in weight, and milk replacement for whey at ratios of 1:0.25, 1:0.538 or 1:1. The processing conditions included a temperature of $90^{\circ} \mathrm{C}$ with an estimated cooking time of 150 minutes, and a final concentration of $79^{\circ} \mathrm{Bx}$.

Keywords: Dulce de leche, whey, dairy dessert, standardization, processing conditions.

\section{INTRODUCCIÓN}

En Colombia, se fabrica una amplia variedad de dulces de leche en los que la técnica de cocción difiere levemente (Lambert, 1998), un ejemplo son las panelitas de leche (PDL) (Cortés et al., 2014). Las PDL o tan solo panelitas son un caramelo duro, obtenido mediante la concentración por evaporación de una mezcla de leche, harina, azúcar y panela, en presencia de un neutralizante como bicarbonato. Dichos caramelos según ICONTEC (2008) son aquellos productos de consistencia maciza, vítrea y de alta concentración de sólidos, que al enfriarse adquieren una consistencia sólida y quebradiza.

Las PDL poseen un color café claro o marrón de intensidad media. Comercialmente, se encuentran variaciones que van desde los tonos muy claros a muy oscuros. Su superficie presenta poca brillantez. Son moderadamente duras, secas y arenosas. De sabor dulce sobresaliente en intensidad moderada, con aroma lácteo. Se comercializan en diferentes presentaciones, similar a un prisma rectangular o cúbico, algunas semiesféricas o de apariencia irregular dependiendo del capricho del artesano o como sea aceptado culturalmente en una determinada región del país (Cortés et al., 2014). En la Tabla 1 se presenta los valores de referencia de la tabla nutricional para PDL (ICBF, 2005).
Tabla 1. Composición nutricional promedio de panelitas de leche.

\begin{tabular}{lcc}
\hline \multicolumn{1}{c}{ Característica } & $\begin{array}{c}\text { Valores de } \\
\text { referencia }\end{array}$ \\
\hline Aporte calórico & $\mathrm{kJ}$ & 1476 \\
Carbohidratos & $\% \mathrm{~m} / \mathrm{m}$ & 83,0 \\
Humedad & $\% \mathrm{~m} / \mathrm{m}$ & 10,6 \\
Contenido de Proteína & $\% \mathrm{~m} / \mathrm{m}$ & 2,9 \\
Materia grasa & $\% \mathrm{~m} / \mathrm{m}$ & 1.8 \\
Ceniza & $\% \mathrm{~m} / \mathrm{m}$ & 1,7 \\
\hline
\end{tabular}

Fuente: ICBF (2005)

En esta investigación se realizaron varios experimentos con el fin de obtener una formulación de PDL con lactosuero. Para ello fue necesario estudiar el efecto de diferentes materias primas y las condiciones del proceso, hasta obtener una formulación de características sensoriales similar a la comercial. Previamente, se obtuvo un perfil sensorial de referencia que sirviera de referencia al momento de evaluar los productos obtenidos en cada experimento.

\section{MATERIALES Y MÉTODOS}

Ubicación. La elaboración, caracterización y análisis sensorial de las diferentes formulaciones de PDL se realizaron en los laboratorios de la Escuela de Ingeniería de Alimentos, Universidad del Valle (Colombia).

Materias primas. Para la elaboración de las muestras de PDL se utilizaron los siguientes productos: leche pasteurizada, leche deslactosada, leche en polvo. lactosuero, harina de arroz, harina de maíz, bicarbonato de sodio, azúcar, panela y glucosa. 
Fabricación de panelitas de leche. Cada día se prepararon aleatoriamente dos lotes de producción (mañana y tarde). Para la fabricación de todas las muestras se siguieron las recomendaciones de Cortés et al. (2014). En la Figura 1 se presenta el diagrama de flujo de elaboración de PDL.

Preparación de la muestra control. Para la elaboración de la muestra control de panelitas de leche se utilizó la siguiente formulación: leche entera $(54,74 \%)$, azúcar $(42,04 \% \mathrm{~m} / \mathrm{m})$, panela $(1,75 \% \mathrm{~m} / \mathrm{m})$, harina arroz $(1,44 \% \mathrm{~m} / \mathrm{m})$, y bicarbonato de sodio de acuerdo con el cálculo aplicando la Eq.1 (aproximadamente 0,03\%m/m).
Previamente a la preparación de las muestras se hidrató la harina de arroz en $25 \mathrm{~mL}$ de leche entera. A la leche entera líquida (relación proteína:grasa, 0,96:1) se le adicionó primero bicarbonato de sodio y panela, calentando y agitando constantemente. Obtenida la solución, se agregó una parte de sacarosa (75\%) y después de su disolución total se añadió harina de arroz. Posteriormente, se adicionó sacarosa restante (25\%). Se agitó constantemente la mezcla y se mantuvo una temperatura entre 105 y $110^{\circ} \mathrm{C}$. Se evaporó la mezcla hasta la concentración deseada $\left(79^{\circ} \mathrm{Bx}\right)$ con un tiempo de cocción aproximado de $90 \mathrm{~min}$. Se enfrió la masa hasta una temperatura de $70^{\circ} \mathrm{C}$ y se moldeó. Finalmente, se cortó y empacó el producto terminado.

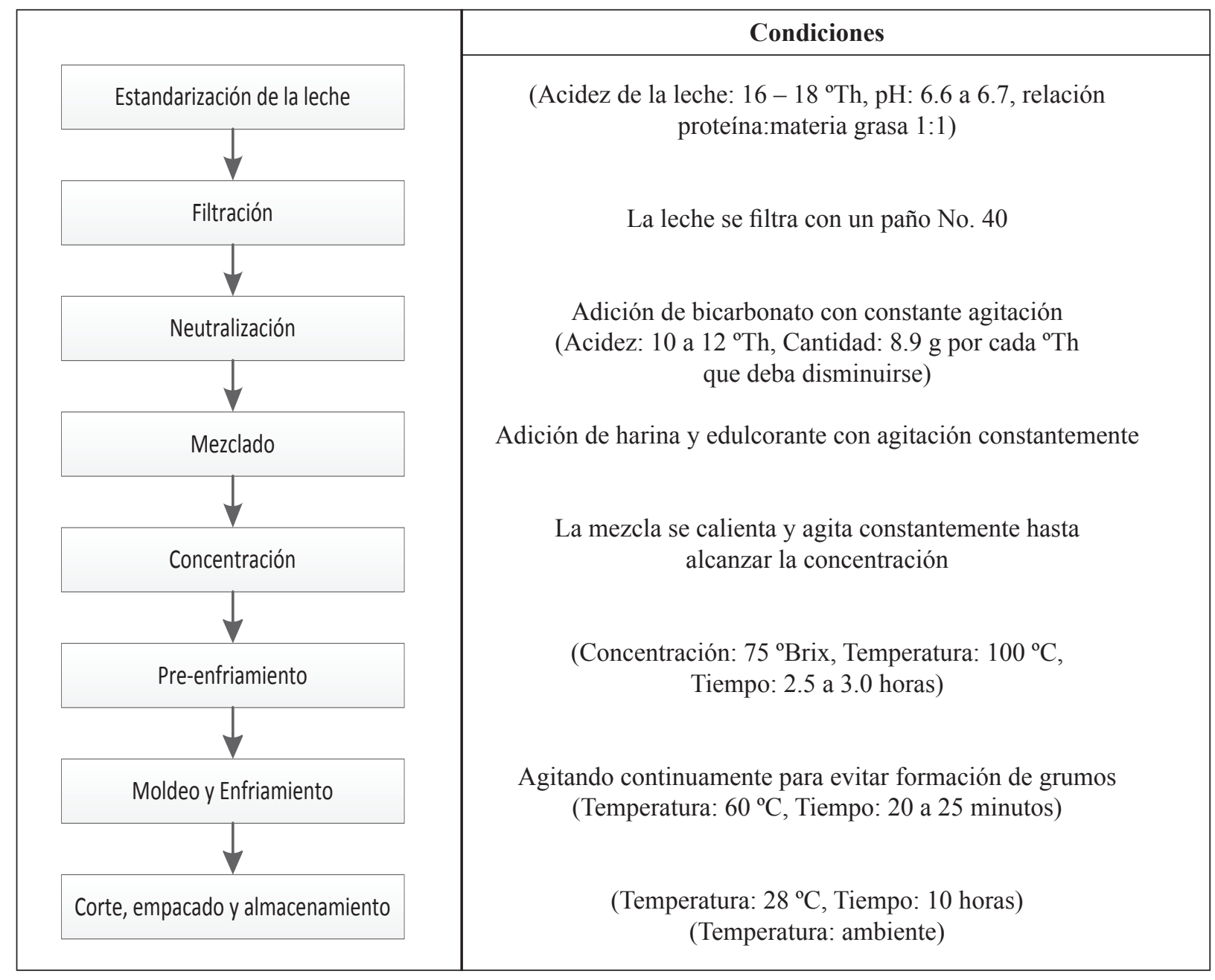

Figura 1. Diagrama de flujo y condiciones de elaboración de panelitas de leche. 
Para el cálculo de la cantidad a adicionar de bicarbonato de sodio se empleó la siguiente ecuación:

$$
m_{\mathrm{NaHCO}_{3}}=\frac{\mathrm{PM}_{\mathrm{NaHCO}_{3}}}{\mathrm{PM}_{\mathrm{C}_{3} \mathrm{H}_{6} \mathrm{O}_{3}}} \frac{V_{L} * \rho_{L} *\left(\% A L_{i}-\% A L_{f}\right)}{\% P_{\mathrm{NaHCO}_{3}}}
$$

Dónde: $\mathrm{m}_{\mathrm{NaHCO}}$ es la masa de bicarbonato de sodio que se debe adicionar para neutralizar la leche. PM son los pesos moleculares del bicarbonato (84g/ gmol) y delácido láctico ( $90 \mathrm{~g} / \mathrm{gmol}) ; \mathrm{V}_{\mathrm{L}}$ es el volumen de leche en $\mathrm{L} ; \rho_{\mathrm{L}}$ es la densidad de la leche en $\mathrm{kg} \cdot \mathrm{L}^{-1}$; $\% \mathrm{AL}_{\mathrm{i}}$ es la acidez inicial de la leche en porcentaje de ácido láctico, determinada por titulación con $\mathrm{NaOH}$ $1 \mathrm{~N}$ y fenolftaleína como indicador; el $\% \mathrm{AL}_{\mathrm{f}}$ final es el valor al que se desea llegar, para este caso fue de $0,12 \% \mathrm{AL} ; \% \mathrm{P}_{\mathrm{NaHCO}}$ es la pureza a la que se encuentre el bicarbonato de sodio.

El contenido de sólidos totales ( ${ }^{\circ} \mathrm{Bx}$ ) se midió utilizando un refractómetro portátil (ATC modelo FG-109, 0-90\%), con compensación de temperatura a $20^{\circ} \mathrm{C}$. Se colocó suficiente muestra para cubrir el lente óptico del equipo y se procedió a leer. La lectura se reportó directamente en la pantalla del equipo. La temperatura se midió con termómetros digitales, introduciendo la lanza directamente en la muestra. La determinación se hizo cada 10 minutos, con el fin de comprobar que la temperatura se mantenía constante.
Diseño experimental. En este estudio preliminar se realizaron ocho experimentos. Tomando como referencia la fórmula original de PDL, se modificó la cantidad empleada de los ingredientes hasta obtener una formulación aceptable a nivel sensorial. En todos los experimentos se aplicaron diseños factoriales. En la Tabla 2, se presentan los factores y niveles de cada experimento. En todos los casos la variable de respuesta principal fue la calidad sensorial. Todos los experimentos se realizaron por duplicado.

Para la elaboración de las muestras en cada uno de los experimentos se siguió el procedimiento descrito para la muestra control, realizando los reemplazos o sustituciones de acuerdo con el experimento.

Experimento 1 - Disolución leche en polvo. Se calculó la cantidad de leche en polvo requerida, con base en los sólidos totales aportados por la leche entera líquida. La leche en polvo se reconstituyó diluyendo en agua (relación 1:1 y 1:0,5). Además de la calidad sensorial se evaluó el tiempo de cocción.

Experimento 2 - Tipo de harina. Con base en la formulación de la muestra control, se elaboraron muestras de PDL utilizando harina de arroz y fécula de maíz. Se comparó la calidad sensorial de las muestras obtenidas.

Tabla 2. Experimentos unifactoriales y los niveles evaluados

\begin{tabular}{cll}
\hline Exp. & \multicolumn{1}{c}{ Factor } & \multicolumn{1}{c}{ Niveles } \\
\hline 1 & Disolución leche en polvo & Dos (agua:leche en polvo, $1: 1,1: 0,5)$ \\
2 & Tipo de harina & Dos (arroz, maíz) \\
3 & Relación edulcorantes & Cuatro (azúcar:glucosa, $1: 0,0256 ; 1: 0,0526 ; 1: 0,0811 ; 1: 0,1111)$ \\
4 & Relación edulcorantes & Tres (azúcar:panela, 1:0,0417; 1:0,087; 1:0,1364) \\
5 & Tipo de leche & Dos (entera, deslactosada) \\
6 & Condiciones de proceso & Dos (temperatura, tiempo) \\
7 & Disolución de lactosuero & Cuatro (agua:lactosuero, $1: 0 ; 1: 1 ; 0,3333: 1 ; 0: 1)$ \\
8 & Cantidad de lactosuero & Seis (leche entera líquida:lactosuero reconstituido, $1: 0 ; 1: 0,25 ;$ \\
& & $1: 0,538 ; 1: 1 ; 0,333: 1 ; 0: 1)$ \\
\end{tabular}


Experimento 3 - Relación edulcorantes. Con base en la formulación de la muestra control, se realizó una sustitución de la azúcar empleada por glucosa líquida (azúcar:glucosa, 1:0,0256; 1:0,0526; 1:0,0811; 1:0,1111).

Experimento 4 - Relación edulcorantes. Con base en la formulación de la muestra control, se realizó una sustitución de la azúcar empleada por panela en bloque (azúcar:panela, 1:0,0417; $1: 0,087 ; 1: 0,1364)$.

Experimento 5 - Tipo de leche. Con base en la formulación de la muestra control, se elaboraron muestras de PDL utilizando leche entera y leche deslactosada. Se comparó la calidad sensorial de las muestras obtenidas.

Experimento 6 - Condiciones de proceso. Con base en los resultados del experimento 5 , se utilizó una concentración de panela del $4 \%$ respecto a la azúcar total empleada. Se varió la temperatura de cocción $\left(90^{\circ} \mathrm{C}, 110^{\circ} \mathrm{C}\right)$. La mezcla se concentró por evaporación hasta $79^{\circ} \mathrm{Bx}$. Además de la calidad sensorial, se evaluó el tiempo requerido para la obtención de la concentración deseada.

Experimento 7 - Disolución de lactosuero. Se calculó la cantidad de lactosuero en polvo requerido, de acuerdo con los sólidos totales aportados por la leche entera líquida. Con base en los resultados del experimento 5 ( $4 \%$ panela) y experimento 6, utilizando una temperatura de cocción de $90^{\circ} \mathrm{C}$. Se realizó el experimento, reemplazando la leche entera líquida por lactosuero dulce disuelto en agua (agua:lactosuero 1:0; 1:1; 0,3333:1; 0:1).

Experimento 8 - Solidos totales lácteos. Con base en los resultados del experimento 5 (4\% panela), experimento $6\left(90^{\circ} \mathrm{C}\right)$ y experimento 7 (agua:lactosuero 1:1), se reemplazó el contenido de leche por lactosuero (leche entera líquida:lactosuero reconstituido, 1:0; 1:0,25; $1: 0,538 ; 1: 1 ; 0,333: 1 ; 0: 1)$

\section{Análisis Sensorial}

Selección del panel. Se convocaron a estudiantes del Programa de Ingeniería de Alimentos de la Universidad del Valle a una reunión en la que se explicó el proyecto. A los participantes se les realizó una encuesta que permitió determinar su disponibilidad de tiempo, interés en el tema, hábitos alimenticios, estado de salud y simpatía hacia el producto. Aquellos que cumplieron positivamente con las preguntas de la encuesta fueron convocados nuevamente para realizar las pruebas básicas (para olor, color y sabor): 1) detección, 2) umbrales y 3) ordenamiento (ISO, 2012). Los panelistas que obtuvieron la mayor calificación fueron seleccionados y convocados posteriormente para el entrenamiento (ICONTEC, 2009a, b; ISO, 2012). Cuatro estudiantes conformaron el panel sensorial (panelistas semientrenados).

Vocabulario de referencia. Con el fin de seleccionar los atributos a evaluar y establecer los términos sensoriales (vocabulario de referencia) se tomó como base la metodología expuesta en la norma AFNOR ISO 11035:1995 (AFN, 1995). Con el fin de adiestrar los sentidos de los catadores frente a este tipo de productos, los panelistas degustaron PDL comerciales provenientes de diferentes empresas ubicadas en diferentes ciudades de Valle del Cauca. Se realizaron ocho sesiones previas (una por semana), donde se cataban las muestras y se realizaban comentarios respecto a sus características sensoriales. A partir de esta actividad se construyó un listado de descriptores, el cual se organizó agrupando los términos semejantes y clasificándolos según el atributo sensorial estudiado. Posteriormente, una vez los panelistas se habían familiarizado con las muestras y conocían diferentes PDL producidas en la región (Valle del Cauca), se realizaron tres sesiones de entrenamiento, donde se explicaron cada uno de los términos de referencia a evaluar. 
Evaluación sensorial de PDL. Se realizaron siete sesiones para la evaluación de las muestras. Se empleó la técnica "Análisis Descriptivo Cuantitativo" (QDA) utilizando una escala no estructurada de $15 \mathrm{~cm}$ con referencias ancladas en $1 \mathrm{~cm}$ (intensidad débil) y/o en $14 \mathrm{~cm}$ (intensidad fuerte) (ICONTEC, 2004; Meilgaard et al., 2007). Al iniciar las sesiones a los estudiantes se les explicó brevemente el objetivo de cada prueba y la forma de percibir las características sensoriales (color, olor, textura y sabor) de cada una de las formulaciones de PDL. Se utilizó un diseño de Bloques Incompletos Balanceados (BIB) (ISO, 2011), a temperatura ambiente $\left(20-23^{\circ} \mathrm{C}\right)$ en platos de material plástico rotulados con números de tres dígitos elegidos al azar. Cada sesión se realizó al día siguiente de la fabricación de los lotes de PDL de cada experimento. Cada evaluador evaluó sólo un subconjunto del número total de muestras en una sola sesión, esto con el fin de evitar la fatiga sensorial y psicológica. A los panelistas se les dio el tiempo necesario para evaluar cada uno de los atributos. El proce- so de evaluación sensorial tuvo una duración entre 70 y 90 min. El subconjunto de muestras que cada evaluador evaluó se seleccionó de modo que, en una sola repetición del diseño BIB, cada muestra se evalúe un número igual de veces y todos los pares posibles de dos muestras se evalúen por un número igual de los evaluadores. Se utilizó análisis de varianza (ANDEVA), como prueba estadística, para analizar los datos de potencia y estadística de Friedman-tipo para clasificar los datos derivados del diseño BIB. Los resultados de cada evaluación sensorial permitieron establecer la formulación para el siguiente experimento.

\section{RESULTADOS Y DISCUSIÓN}

Vocabulario sensorial. Con el panel semientrenado se estableció el vocabulario de referencia (Tabla 3) que se utilizó en la evaluación de las muestras de PDL de los diferentes experimentos. También se construyó un perfil sensorial de referencia (Figura 2).

Tabla 3. Vocabulario sensorial para evaluación de panelitas de leche.

\begin{tabular}{|c|c|}
\hline Descriptor & Definición \\
\hline Amargo & $\begin{array}{l}\text { Sabor indeseable amargo, producido por presencia de concentraciones no homogenizadas y } \\
\text { sectorizadas de lactosuero dentro de la matriz alimentaria. }\end{array}$ \\
\hline Arenoso & $\begin{array}{l}\text { Sensación que se aprecia en la cavidad bucal posterior a la masticación y que se percibe } \\
\text { debido a la presencia de fragmentos muy pequeños. }\end{array}$ \\
\hline Betas blancas & $\begin{array}{l}\text { Imperfección en el alimento en forma de betas blancas (leneas irregulares) y que hace perder } \\
\text { homogeneidad en el color del alimento. }\end{array}$ \\
\hline Caramelo & $\begin{array}{l}\text { Sensación olfativa y gustativa producida por la polimerización de los azúcares cuando son } \\
\text { tratados a altas temperaturas. }\end{array}$ \\
\hline Compacto & $\begin{array}{l}\text { Sensación, percibida al tacto, debido a la resistencia de las partículas del mismo alimento a } \\
\text { dejarse fragmentar en unidades más pequeñas al aplicarse una fuerza. }\end{array}$ \\
\hline Crema (grasa láctea) & $\begin{array}{l}\text { Sabor y aroma característico a grasa láctea que se puede percibir en productos que contienen } \\
\text { leche. }\end{array}$ \\
\hline Dulce & $\begin{array}{l}\text { Sensación básica olfativa y gustativa, percibida ante todo en las de la lengua, que se asocia a } \\
\text { la presencia de edulcorantes. }\end{array}$ \\
\hline Duro & $\begin{array}{l}\text { Sensación de resistencia a la penetración de los dientes ofrecida por parte del alimento al } \\
\text { momento de llevar a cabo la mordida o masticación. }\end{array}$ \\
\hline Friable & $\begin{array}{l}\text { Capacidad para fracturarse con facilidad en pequeñas piezas de forma irregular cuando se } \\
\text { frota con los dedos. }\end{array}$ \\
\hline Intensidad de marrón & $\begin{array}{l}\text { Tonalidades de color marrón que tiene como particularidad, ser de intensidad suave, similar } \\
\text { al de la bebida café en leche. }\end{array}$ \\
\hline Lácteo & $\begin{array}{l}\text { Sabor y aroma característico a producto lácteo que se puede percibir en productos que } \\
\text { contienen leche. }\end{array}$ \\
\hline
\end{tabular}


Continuación: Tabla 3.

\begin{tabular}{|c|c|}
\hline Descriptor & Definición \\
\hline Leche condensada & $\begin{array}{l}\text { Sabor y aroma de grasa láctea, junto con el producto de la reacción de Maillard, que, en } \\
\text { simultáneo, tienen olor de la leche que ha sido sometida a reducción bajo temperatura con } \\
\text { adición de azúcar. }\end{array}$ \\
\hline Mohoso & $\begin{array}{l}\text { Aroma y sabor indeseable de las panelitas de leche, característico a moho que se desarrolla } \\
\text { por el inadecuado manejo de la humedad durante el almacenamiento. }\end{array}$ \\
\hline Panela & $\begin{array}{l}\text { Sensación olfativa y gustativa intensa asociada a la miel de panela, indeseable en las panelitas } \\
\text { de leche. }\end{array}$ \\
\hline Particulado & $\begin{array}{l}\text { Sensación que se aprecia en la cavidad bucal posterior a la masticación y que se percibe debido } \\
\text { a la presencia de fragmentos un poco más grandes que los gránulos de sensación arenosa. }\end{array}$ \\
\hline Quebradizo & $\begin{array}{l}\text { Sensación que se aprecia al tacto y que se manifiesta en la fragmentación del alimento instantes } \\
\text { después de aplicársele una fuerza suficiente para que esta seda. }\end{array}$ \\
\hline Químico & $\begin{array}{l}\text { Aroma y sabor a compuestos químicos como desinfectantes, sales minerales }(\mathrm{Cu}, \mathrm{Fe}, \mathrm{Mg}) \mathrm{o} \\
\text { fármacos, indeseables en panelitas de leche. }\end{array}$ \\
\hline Recalentado & $\begin{array}{l}\text { Aroma y sabor indeseables a caramelo que se desarrollan en el alimento al hervirse, por un } \\
\text { calentamiento prolongado, perdiéndose en la panelita de leche los compuestos de aroma y } \\
\text { sabor característicos. }\end{array}$ \\
\hline $\begin{array}{l}\text { Reflexión de la luz en } \\
\text { superficie }\end{array}$ & $\begin{array}{l}\text { Sensación visual que se produce por la cantidad de luz que el alimento tiene la capacidad de } \\
\text { absorber o reflejar en la superficie y da el aspecto opaco o brillante. }\end{array}$ \\
\hline Seca & $\begin{array}{l}\text { Sensación que se aprecia en la cavidad bocal, y que generara sensación de ausencia de } \\
\text { humedad, sed o necesidad de consumo de líquido insípido. }\end{array}$ \\
\hline
\end{tabular}



Figura 2. Perfil sensorial de panelitas de leche. 
Con base en el vocabulario y el perfil sensorial de referencia de PDL se realizó la evaluación de cada formulación. Varias formulaciones fueron rechazadas por el panel sensorial debido a que presentaban características indeseables o marcadas diferencias con respecto al perfil sensorial de referencia de PDL. Con la información suministrada por el panel sensorial se seleccionó la formulación y procedimiento deseado.

\section{Experimentos}

Experimento 1 - Disolución leche en polvo. En las muestras analizadas se observaron tonalidades finales de marrón muy claras y la formación de betas, resequedad perceptible al tacto y sensación bucal muy arenosa, con tamaño de cristal grande y difícil de masticar. Se evidenció la carencia del sabor y olor característico de PDL.

Experimento 2 - Tipo de harina. No se observaron diferencias sensoriales significativas entre las muestras de PDL elaboradas con harina de arroz y fécula de maíz. La textura de los dos tipos de panelitas fue similar. En las muestras se evidenció el color, olor y sabor característico de las PDL.

Experimento 3 - Relación edulcorantes. En todas las formulaciones se observó que la glucosa cumple la función de evitar la rápida cristalización de los azúcares de las PDL. Sin embargo, en concentraciones mayores a 2,5\% de sustitución de azúcar por glucosa, no se logró la solidificación y compactación requerida. Los productos obtenidos presentaban características similares a las del arequipe, aunque el color tenía tonalidades de marrón muy débiles. La textura observada fue similar a la descrita por Maldonado y Guiado (2009), característica de un caramelo blando de leche tipo toffee. A mayor concentración de glucosa, mayor plasticidad en las muestras. Aunque la glucosa aporta diversas características, como aportar dulzura, equilibrio adecuado de azúcares en las formulaciones, control de cristalización, brillo, maquinabilidad y textura (Vermeulen et al., 2014), las texturas obtenida en este experimento no son la adecuadas para una PDL.

Experimento 4 - Relación edulcorantes. Las PDL $(1: 0,087 ; 1: 0,1364)$ obtenidas presentaron textura y color más cercano al perfil sensorial de referencia. Sin embargo, la panela aporta un sabor característico que aleja el producto del sabor de referencia. La muestra con una sustitución del 4\% (1:0,0417) presentó características texturales similares al perfil sensorial de referencia. Obtuvo tonos marrón similares a las PDL comerciales y el sabor a panela de caña fue imperceptible. En las siguientes formulaciones se adoptó la sustitución de azúcar por panela de caña del 4\%. La adición de panela a la formulación aporta textura y color. Esto se debe a la presencia de azúcar reductor en forma de glucosa en la composición de la panela (Boutin, 2000).

Experimento 5 - Tipo de leche. En las muestras elaboradas con leche deslactosada se observaron tonalidades marrón oscuras indeseadas, acompañadas de sabores y aromas a quemado. Se cree que este incremento se debe a que la lactosa hidrolizada tiene un poder reductor superior al que presenta en estado nativo y a que la leche empleada tiene mayor cantidad de azúcares reductores aportados por la glucosa y la galactosa (Barbosa et al., 2007), esto favorece la reacción de Maillard.

Experimento 6 - Condiciones de proceso. Se observó que las muestras producidas a temperaturas de $90^{\circ} \mathrm{C}$ requirieron mayor tiempo para alcanzar la concentración final. El producto obtenido presentó características deseables y aceptables en todos los atributos evaluados sensorialmente. Keating y Rodriguez (1999) expresan que la cocción en confites debe ser larga y sostenida, propiciando un mejor desarrollo de las diferentes reacciones e interacciones de las materias primas involucradas en el proceso para incrementar la calidad del producto final. 
Experimento 7 - Disolución de lactosuero. De los resultados obtenidos en los diferentes grados de disolución (agua:lactosuero 1:0; 1:1; 0,33:1; 0:1) se observó que las disoluciones al 1:0 y 1:1 empleadas en la fabricación de PDL presentaron características muy similares a las obtenidas en el experimento anterior. Sin embargo, las muestras elaboradas con disoluciones 0,33:1 y 0:1 presentaron características de cristal muy grande y difícil de masticar, esto puede deberse al bajo contenido de humedad en la mezcla inicial. Adicionalmente, se cree que la lactosa aportada por el lactosuero favorece la recristalización rápida que afecta las propiedades texturales. Para el experimento posterior se seleccionó la disolución 1:1.

Experimento 8 - Solidos totales lácteos. Se observó que en los reemplazos mayores al 1:1 se presentaron muestras con mucha dureza, difíciles de cortar y masticar. Además se evidenció la formación de tonalidades blancas en la superficie de los productos. Estas características se deben posiblemente a la alta cantidad de sólidos presentes y la cristalización de la lactosa, que en altas concentraciones forma rápidamente un cristal compacto y de gran tamaño que incrementa la dureza (AOAC, 2003; Codex-289, 2011; Pereyra Gonzales et al., 2010).

Características sensoriales. Una PDL ideal es aquella que presenta características aceptables de tamaño del cristal, textura y sabor; adecuado equilibrio en la cantidad de agua respecto a los azúcares en la matriz del alimento. Al fabricar las PDL con harina de arroz o con fécula de maíz se observó que es importante disolver adecuadamente éstas antes de adicionarlas a la leche. Caso contrario la textura de las PDL se ve afectada por la formación de grumos.

La tonalidad marrón se debe a la reacción de Maillard, que tiene estrecha relación con la relación tiempo:temperatura y azúcar:proteína. La reacción de Maillard se inicia a partir de la condensación de grupos carbonilo y amino seguido por un conjunto de reacciones complejas que ocurren en los alimentos durante el calentamiento (Ramírez-
Navas, 2011), y que se intensifica debido al aumento en gran medida de los azúcares reductores capaces de entrar en contacto con los grupos aminos presentes en las proteínas de la leche.

Un adecuado manejo de estas relaciones permite obtener adecuadas características sensoriales (sabor, aroma y color) en los productos finales (Ramírez-Navas, 2011). Una cocción prolongada a temperaturas cercanas a $90^{\circ} \mathrm{C}$ permite conseguir estas características en el producto final. Empleo de tiempos cortos a altas temperaturas son la causa directa de la carencia de los atributos mencionados anteriormente, tonalidades débiles de marrón y aroma débil. Sin embargo, tiempos demasiado prolongados causa el oscurecimiento innecesario de la muestra.

Por otra parte, la relación de agua:sólidos totales tienen gran impacto sobre la textura. Por ejemplo, bajas concentraciones de agua produce PDL con alta dureza. Si entre los sólidos existe un alto contenido de lactosa, la recristalización es muy rápida y las PDL presentan un cierto grado de arenosidad. También la caramelización de la sacarosa tiene influencia directa en el estado cristalino final del producto (Keating et al., 1999).

\section{CONCLUSIONES}

Se pueden obtener PDL similares a las comerciales utilizando harina de maíz, sustituyendo de 4\% de panela por azúcar, con lactosuero diluido al $50 \%$ en peso y proporciones de reemplazo de leche por lactosuero del 1:0,25; 1:0,538 o 1:1. Las condiciones del proceso fueron: temperaturas de $90^{\circ} \mathrm{C}$, tiempo de cocción de 150 minutos y concentración final de $79^{\circ} \mathrm{Bx}$. La intensidad de tonalidad marrón en el producto final tiene relación directa con el tiempo de cocción.

\section{AGRADECIMIENTOS}

Los autores agradecen a la señora Sorayda Parra Tribiño, propietaria de micro empresa de dulces La Panchita (Roldanillo, Valle del Cauca, Colombia), por explicar el proceso de elaboración de las 
panelitas de leche. A Martha C. Viveros y Scott Pettit por su colaboración con la revisión del texto en inglés. Y a las autoridades y personal de la Escuela de Ingeniería de Alimentos de la Universidad del Valle por toda la colaboración brindada.

\section{REFERENCIAS BIBLIOGRÁFICAS}

AFNOR-ISO. ASSOCIATION FRANÇAISE DE NORMALISATION. 1995. NF-ISO-11035. Sensory Analysis - Identification and Selection of Descriptors for Establishing a Sensory Profile by a Multidimensional Approach. Association Francaise de Normalisation, Paris, Francia. 26 p.

AOAC. ASSOCIATION OF OFfICIAL ANALYTICAL CHEMISTS. 2003. AOAC-920.39 - Official Methods of Analysis: Diethylether Extraction-Submersion. Association of Official Analytical Chemists, Gaithersburg, USA. 1 p.

BARBOSA, G.; SCHMIDT, S.J.; FONTANA, A.J.; LABUZA, T.P. 2007. Water Activity in Foods: Fundamentals and Applications. Institute of Food Technologists - Wiley-Blackwell. $440 \mathrm{p}$.

BOUTIN, R. 2000. Confections: History and Product Categories. En: NAKAI S Y H. Food Proteins: Processing Applications. Wiley-VCH, Inc, Canada. 536 - 539 p.

CODEX-289. 2011. Sueros en polvo. Codex alimentarius. Food and Agriculture Organization. Roma. Italia. 4 p.

CORTÉS, A.; ORTIZ, J.R.; RAMÍREZ-NAVAS, J.S. 2014. Panelitas de leche colombianas. Tecnol. Láctea Latinoam. 81(1):52 - 61.

ICBF. 2005. Tabla de Composición Nutricional. En: Instituto Colombiano de Bienestar Familiar. http://alimentoscolombianos.icbf.gov.co/alimentos_colombianos/; consultada: febrero, 2015.

ICONTEC. INSTITUTO COLOMBIANO DE NORMAS TECNICAS Y CERTIFICACION. 2004. NTC5328-Análisis sensorial. Directrices para el uso de escalas de respuesta cuantitativas. Instituto Colombiano de Normas Técnicas y Certificación, Bogotá, Colombia. 15 p.

ICONTEC. INSTITUTO COLOMBIANO DE NORMAS TÉCNICAS Y CERTIFICACIÓN. 2008. NTC424-Productos Alimenticios. Caramelos Duros Instituto Colombiano de Normas Técnicas y Certificación, Bogotá, Colombia. 9 p.

ICONTEC. INSTITUTO COLOMBIANO DE NORMAS TÉCNICAS Y CERTIFICACIÓN. 2009a. GTC178-1-Análisis sen- sorial. Guía general para el personal de un laboratorio de evaluación sensorial. Parte 1: responsabilidad del personal. Instituto Colombiano de Normas Técnicas y Certificación, Bogotá, Colombia. 16 p.

ICONTEC. INSTITUTO COLOMBIANO DE NORMAS TÉCNICAS Y CERTIFICACIÓN. 2009b. GTC178-2-Análisis sensorial. Guía general para el personal de un laboratorio de evaluación sensorial. Parte 1: reclutamiento y formación de líderes de panel, Bogotá, Colombia. 19 p.

ISO. INTERNATIONAL ORGANIZATION FOR STANDARDIZATION. 2012. IS08586-Sensory analysis - General guidelines for the selection, training and monitoring of selected assessors and expert sensory assessors. International 0rganization for Standardization, Geneva, Switzerland. $28 \mathrm{p}$.

KEATING, P.F.; RODRÍGUEZ, H.G. 1999. Introducción a la lactología. Editorial Limusa S.A. De C.V., Monterrey, México. $316 \mathrm{p}$.

LAMBERT, E. 1998. Cocina Latinoamericana: Más de 250 recetas de las más sabrosas de los países americanos desde México a la Patagonia. Editorial Edaf, San Juan, Puerto Rico. 416 p.

MALDONADO, R.; GUIADO, M. 2009. Elaboración de caramelo blando de leche (tipo toffee) a partir de lactosuero deshidratado. Rev. Fac. Agronom. Univ. Central Venezuela. 35(1):1 - 7 .

MEILGAARD, M.; CIVILLE, G.V.; CARR, B.T. 2007. Sensory evaluation techniques. 4th ed. Taylor \& Francis, Boca Raton, USA. 448 p.

PEREYRA-GONZALES, A.S.; NARANJO, G.B.; LEIVA, G.E.; MALEC, L.S. 2010. Maillard reaction kinetics in milk powder: Effect of water activity at mild temperatures. Int. Dairy J. 20(1):40 - 45. http://dx.doi.org/10.1016/j. idairyj.2009.07.007.

RAMÍREZ-NAVAS, J.S. 2011. Estudio del efecto de la fortificación con hierro y suplementación con lactosuero en polvo sobre algunas propiedades funcionales, físicas y sensoriales de un queso de pasta cocida. Universidad del Valle, Cali, Colombia. $110 \mathrm{p}$.

VERMEULEN, A.; MARVIG, C.L.; DAELMAN, J.; XHAFERI, R.; NIELSEN, D.S.; DEVLIEGHERE, F. 2014. Strategies to increase the stability of intermediate moisture foods towards Zygosaccharomyces rouxii: The effect of temperature, ethanol, $\mathrm{pH}$ and water activity, with or without the influence of organic acids. Food Microb. 45(A):119-125. http://dx.doi.org/10.1016/j.fm.2014.01.003. 